\title{
Extent of the low-velocity region in the lowermost mantle beneath the western Pacific detected by the Vietnamese Broadband Seismograph
}

\section{Array}

\author{
N. Takeuchi, ${ }^{1}$ Y. Morita, ${ }^{1}$ N. D. Xuyen, ${ }^{2}$ and N. Q. Zung ${ }^{2}$ \\ Received 7 January 2008; revised 20 January 2008; accepted 6 February 2008; published 11 March 2008.
}

[1] We present evidence showing the extent of the lowvelocity region in the lowermost mantle beneath the western Pacific. We analyzed $S, s S, S c S-S$, and $s S c S-s S$ travel times observed by the Vietnamese broadband seismograph array deployed as part of the Ocean Hemisphere Project. The abrupt changes in $S c S-S$ and $s S c S-s S$ travel times suggest that the western geographical boundary of the low-velocity region is located around $140^{\circ} \mathrm{E}$ and is sharp (more than $4 \%$ velocity contrast within $200 \mathrm{~km}$ ). The dependency of $S$ and $s S$ travel time anomalies of epicentral distances suggests that the strong low-velocity region is confined to within $400 \mathrm{~km}$ from the CMB (core-mantle boundary). The existence of lateral heterogeneities with a $100 \mathrm{~km}$ scale inside the low-velocity region is also suggested by the abrupt changes in $S$ and $S c S$ waveforms. Citation: Takeuchi, N., Y. Morita, N. D. Xuyen, and N. Q. Zung (2008), Extent of the low-velocity region in the lowermost mantle beneath the western Pacific detected by the Vietnamese Broadband Seismograph Array, Geophys. Res. Lett., 35, L05307, doi:10.1029/ 2008GL033197.

\section{Introduction}

[2] In most whole-mantle tomographic models, we see two LLVPs (Large Low-Velocity Provinces) beneath Africa and the Pacific in the lowermost mantle. Elucidation of the similarities and differences between these LLVPs should give further clues regarding the thermal and chemical structures of the CMB region, and such structures should provide important constraints on the dynamics of mantle upwellings.

[3] Extensive studies have been conducted to reveal the fine velocity structure of the African LLVP [e.g., Ritsema et al., 1998; Ni et al., 2002]. It has been suggested that the African LLVP, clearly bounded by sharp side edges, has a low-velocity region extending for about $1300 \mathrm{~km}$ from the CMB. On the other hand, the extent of the Pacific LLVP is relatively unknown, especially beneath the western Pacific.

[4] Takeuchi [2007] conducted whole-mantle waveform tomography, which suggested that the strong low-velocity region beneath the Pacific is confined to within $300-400 \mathrm{~km}$ from the CMB. However, the spatial resolution of this study is limited because the model was obtained by inverting longer period waves (the periodic range used as the data set

\footnotetext{
${ }^{1}$ Earthquake Research Institute, University of Tokyo, Tokyo, Japan.

${ }^{2}$ Institute of Geophysics, Vietnamese Academy for Science and Technology, Hanoi, Vietnam.
}

Copyright 2008 by the American Geophysical Union. 0094-8276/08/2008GL033197\$05.00 was 50-400 sec). He et al. [2006] conducted waveform modeling of the $S c S$ precursor as well as $S c S-S$ travel time analysis, and they suggested $12-13 \%$ velocity reductions in the lowermost $220 \mathrm{~km}$ mantle in the northwestern part of the Pacific LLVP. However, this model was obtained under the assumption of the existence of an ultra-low-velocity $(-13 \%)$ basal layer. Because of the limited resolution of their data set for revealing the vertical dependency of velocity structure, the model appears to be dependent on this assumption.

[5] The purpose of this study is to constrain the lateral and vertical extents of the Pacific LLVP beneath the western Pacific. We analyze the data from the Vietnamese broadband seismograph array deployed as part of the Ocean Hemisphere Project (http://eri-ndc.eri.u-tokyo.ac.jp). The array has a longer dimension in the direction to the FijiTonga region (see Figure 1), and the data from this array provide $S$ and $s S$ for various epicentral distances (the maximum epicentral distance is about $93^{\circ}$ ). We can thus analyze $S$ and $s S$ with various bottoming depths (the greatest bottoming depth is about $150 \mathrm{~km}$ from the CMB), and some of them should be inside the LLVP. These data should provide unique opportunities especially to reveal the vertical extent of the LLVP. After identifying the western geographical boundary, we obtain an average 1D velocity model of the LLVP. The existence of the lateral heterogeneities inside the LLVP are also explored.

\section{Geographical Boundary of the Pacific LLVP}

[6] We first define the western geographical boundary of the Pacific LLVP from $S c S-S$ and $s S c S-s S$ travel times. In the analyses in this and the next section, we use data from the six stations of the Vietnamese broadband seismograph array (blue circles in Figure 1) together with two nearby IRIS GSN stations (KMI and QIZ; light blue circles in Figure 1). We use data for events that occurred in 20012005 , the period when these eight stations worked as a seismograph array network.

[7] We used the transverse component of displacement waveforms to measure the travel times. We applied a high pass filter with a corner frequency of $1 / 100 \mathrm{~Hz}$ to reduce lower-frequency noise. We picked the peak time of the displacement wavepackets for $S, S c S, s S$, and $s S c S$ to obtain $S c S-S$ and/or $s S c S-s S$ travel times. We discarded data that appeared to be highly contaminated by others phases (e.g., $S c S$ and $s S c S$ for larger distances). The epicentral distances of the resultant data are $65-84^{\circ}$. Note that contaminations by $S d S$ are considered to be small because no clear $S d S$ is observed in our data set. We obtained $22 S c S-S$ travel times and $13 s S c S-s S$ travel times for 11 deep events (more than 


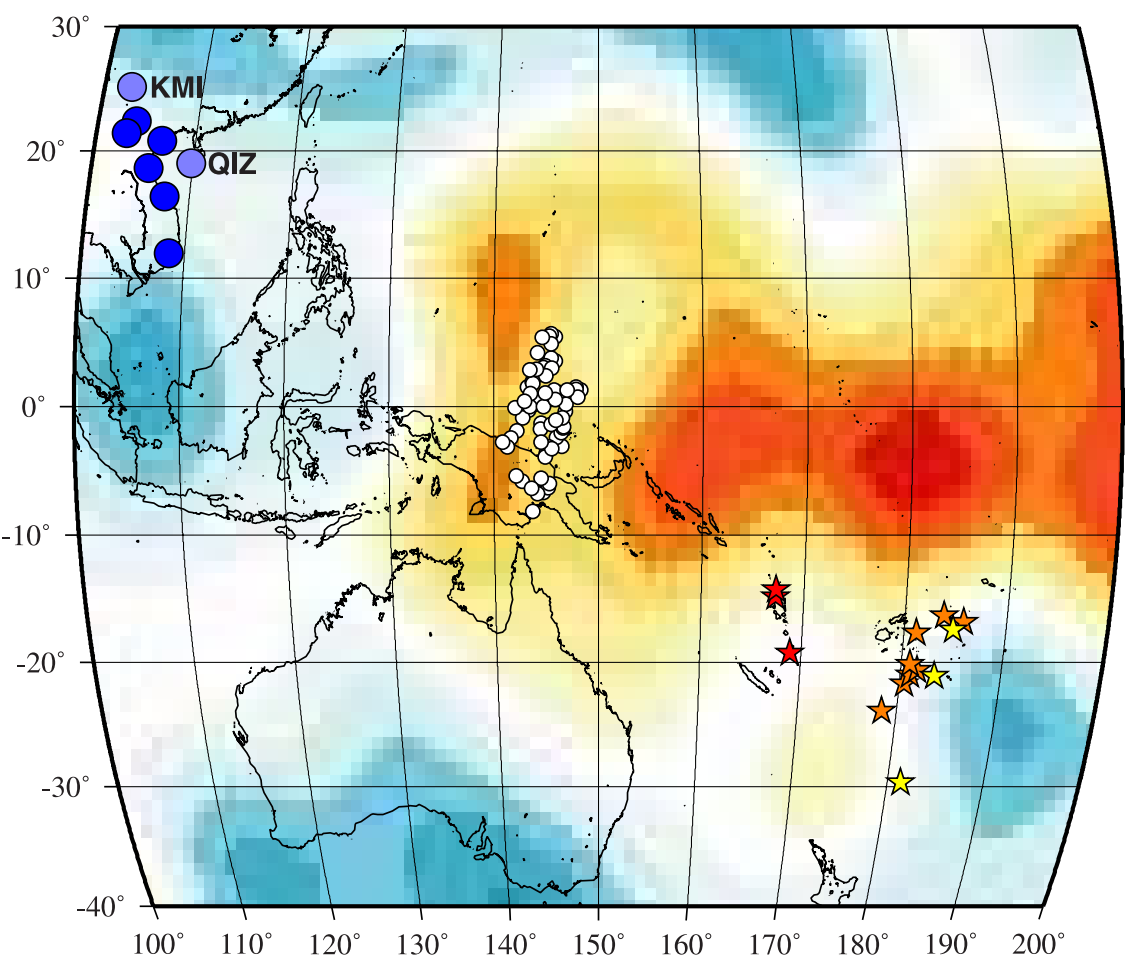

Figure 1. Distribution of events and stations used in data analyses in this study (stars and large circles, respectively). Red and orange stars show events whose $S c S-S$ and/or $s S c S-s S$ travel time data are used in our analyses. Orange and yellow stars show events whose $S$ and/or $s S$ travel time data are used. Dark and light-blue circles show stations of the Vietnamese broadband seismograph array and nearby IRIS GSN stations, respectively. White circles show the $S$ and $s S$ bottoming points of our data set. The heterogeneity pattern at the CMB of the global SH model, SH18CE [Takeuchi, 2007], is overplotted.

$100 \mathrm{~km}$ depth; shown in Figure 1). The observed travel times are compared to the synthetic travel times for the IASP91 structure model [Kennett and Engdahl, 1991] and PDE source parameters.

[8] The results are plotted in Figure 2a. Systematic differences between the west of $139^{\circ} \mathrm{E}$ and the east of $142^{\circ} \mathrm{E}$ are observed: the east shows very large positive travel time anomalies, whereas the west shows small anomalies. This indicates that the western geographical boundary of the Pacific LLVP is around $140^{\circ} \mathrm{E}$. The observed abrupt changes (about $5 \mathrm{~s}$ ) of travel time anomalies within $200 \mathrm{~km}$ indicate that the western edge of the Pacific LLVP is sharp. The geographical location of the boundary is generally consistent with those obtained by previous studies using $S c S-S$ and $s S c S-s S$ travel times [Wysession et al., 1994; He et al., 2006]. The location is also consistent with that indicated by To et al. [2005], who analyzed $S_{\text {dif }}$ travel times: they detected a rapid $S_{\text {dif }}$ travel time shift with respect to the back azimuth at the YAK station $\left(62.03^{\circ} \mathrm{N}, 129.68^{\circ} \mathrm{E}\right)$ for events in the MacquarieBalleny islands region.

\section{Vertical Extent of the Pacific LLVP}

[9] In this section, we constrain the vertical extent of the Pacific LLVP by obtaining a velocity model to fit $S, s S, S c S$ $S$, and $s S c S-s S$ travel time data. Although the purpose of this modeling is to constrain the average $1 \mathrm{D}$ velocity structure of the LLVP, the results in Figure 2a intuitively illustrate the necessity for introducing, at least, 2D velocity model. We thus assume a $2 \mathrm{D}$ velocity model that allows lateral heterogeneities only between the LLVP region and the rest of the region. The geographical boundary between these two regions was constrained by the results in the previous section. However, due to the limitations of the coverage of our data set, the strike of the boundary is not well constrained. In the previous studies, the model by $\mathrm{He}$ et al. [2006] indicated the boundary in the NE-SW direction, whereas To et al. [2005] preferred the boundary in the N-S direction. The region suggested by To et al. [2005] is close to our studied region, and the sampling of the data set of $\mathrm{He}$ et al. [2006] is poor in this region. In the analyses below, we thus assume that the strike of the boundary is in the N-S direction. The detailed location of the boundary is also ambiguous between $139^{\circ} \mathrm{E}$ and $142^{\circ} \mathrm{E}$. We thus test three cases in which the boundary is located at $139^{\circ} \mathrm{E}, 140.5^{\circ} \mathrm{E}$, and $142^{\circ} \mathrm{E}$, and we call our preferred models for these three cases Model A, Model B, and Model C, respectively.

[10] The data used are $S c S-S$ and $s S c S-s S$ travel times (shown in Figure 2a) and travel times of $S$ and $s S$ sampling the LLVP region (50 $S$ travel times and $24 s S$ travel times for 11 events; shown in Figure 1). The reference source and structure models for measuring the travel time anomalies are the same as those described in the previous section. Absolute $S$ and $s S$ travel time data are considered to be contaminated by the errors in the source parameters and the heterogeneities outside of the region of interest. To reduce the effects of these contaminations, we subtract the aver- 
(a)

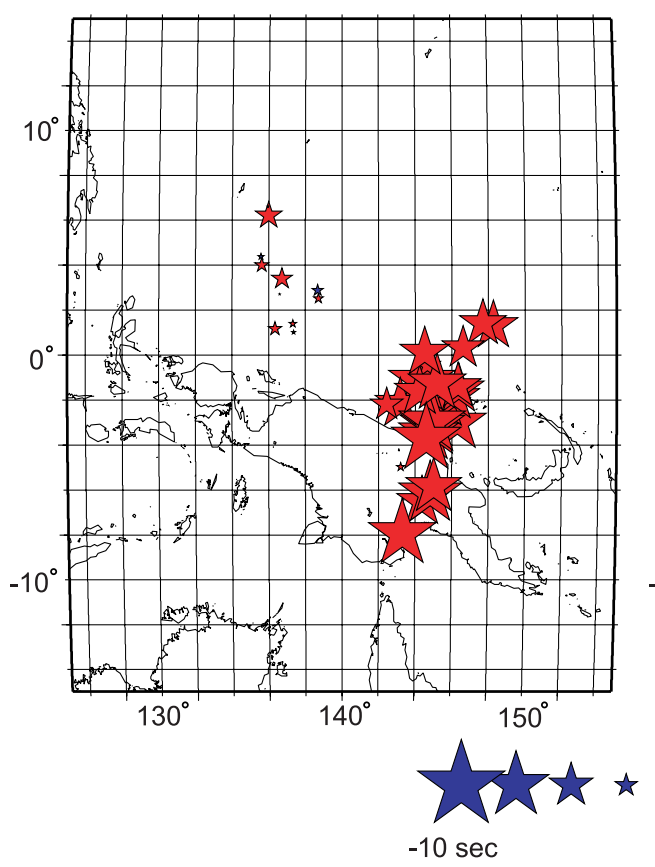

(b)

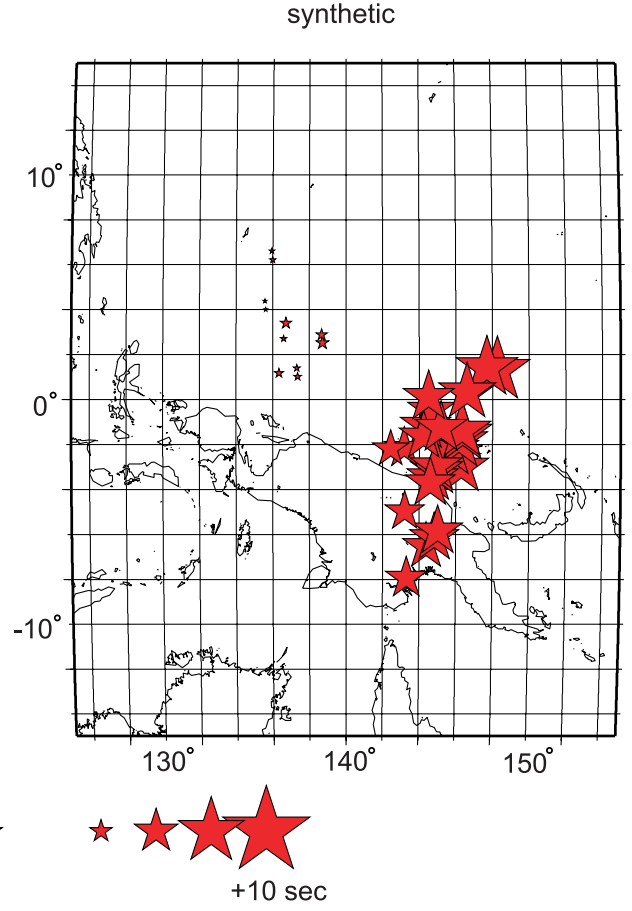

Figure 2. (a) Observed $S c S-S$ and $s S c S-s S$ travel time residuals projected at the bouncing points of $S c S$ or $s S c S$. Red and blue stars indicate positive and negative residuals, respectively, and the sizes of the stars show the absolute quantity of the anomaly. (b) The same as Figure 2a except that synthetic $S c S-S$ and $s S c S-s S$ travel time residuals computed for Model B (shown in Figure 3a) are plotted.

aged travel time residuals (for all stations used) from the observed $S$ and $s S$ travel times.

[11] The observed travel time residuals of $S$ and $s S$ show clear trends in relation to the bottoming depth (red circles in Figure $3 \mathrm{~b}$ ): $S$ and $s S$ data, whose bottoming depth is within $400 \mathrm{~km}$ from the CMB, gradually increase as the bottoming depth increases. This indicates that the strong low-velocity region is confined in the lowermost $400 \mathrm{~km}$. Note that the bottoming depths shown in this paper are those for the IASP91 model and are not necessarily those for the actual Earth structure. They are used just as indices to show epicentral distances with source depth corrections. Note also that we refer to individual source depths and epicentral distances rather than to bottoming depths in obtaining velocity models, and that the results in this paper are not biased by these approximated indices.

[12] Figures $3 \mathrm{a}$ and $3 \mathrm{~b}$ show our preferred models. We tested LLVP models with different thickness and velocity gradient and chose the model best fitting the observed data. The models are obtained by using 2D ray tracing, which rigorously takes nonlinearity into account. We do not see significant differences among Models A, B, and C in the variance reductions. The variance reductions for $S c S-S$ and $s S_{C} S-s S$ data are $91.0 \%, 91.2 \%$, and $91.4 \%$ for Models A, B, and C, respectively, and those for $S$ and $s S$ data are $64.2 \%$, $64.1 \%, 64.0 \%$, respectively. The strong low-velocity region (red or orange in Figure $3 \mathrm{~b}$; less than $-1.265 \%$ ) is confined to within the lowermost $400 \mathrm{~km}$, as was expected from the observed data. Note that the color palette in Figure $3 \mathrm{~b}$ and the definition of strong velocity anomalies follow those of
Takeuchi [2007]. More than 4\% velocity contrast is introduced in the lowermost $200 \mathrm{~km}$.

[13] The models quantitatively explain the observed travel time residuals. The synthetic $S$ and $s S$ residuals are within the scatter of the observed data for every distance (Figure 3c; presented only for Model B). The models also explain the large contrast of $S c S-S$ and $s S c S-s S$ travel time residuals between the eastern and western regions (Figure 2; presented only for Model B): the observed and synthetic average travel time residuals are both $0.8 \mathrm{~s}$ when the bouncing point is west of the geographical boundary of the LLVP and $5.8 s$ when the point is east of the boundary.

\section{Lateral Heterogeneities Inside the LLVP}

[14] In the above analyses, we assumed lateral homogeneity inside the LLVP. In this section we show evidence for the existence of lateral heterogeneities inside the LLVP and their scale lengths. Figure 4 a shows the travel time residual data of $S$ whose bottoming depth is within $300 \mathrm{~km}$ from the CMB. We see four bottoming points (in the thick black square in Figure 4a) located very close to each other but with greatly differing travel time residuals. The southernmost bottoming point (for the event on $08 / 19 / 2002$, labeled 020819) has a large positive residual, whereas the northern three bottoming points (for the events labeled 021004, 021022, 040111) have almost no residuals. These four data are all from the same station (KMI from IRIS GSN), and their bottoming depths are almost identical $(256 \mathrm{~km}, 243 \mathrm{~km}$, $244 \mathrm{~km}$, and $251 \mathrm{~km}$, respectively). These bottoming points 
(a)

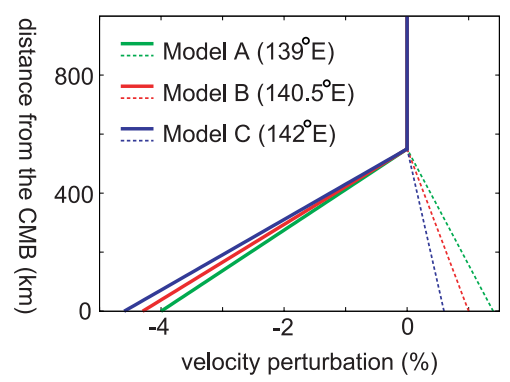

(b)

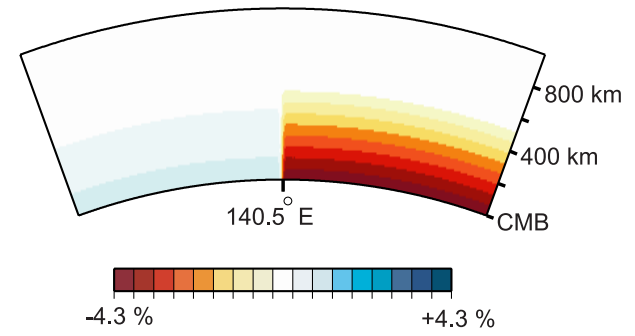

(c)

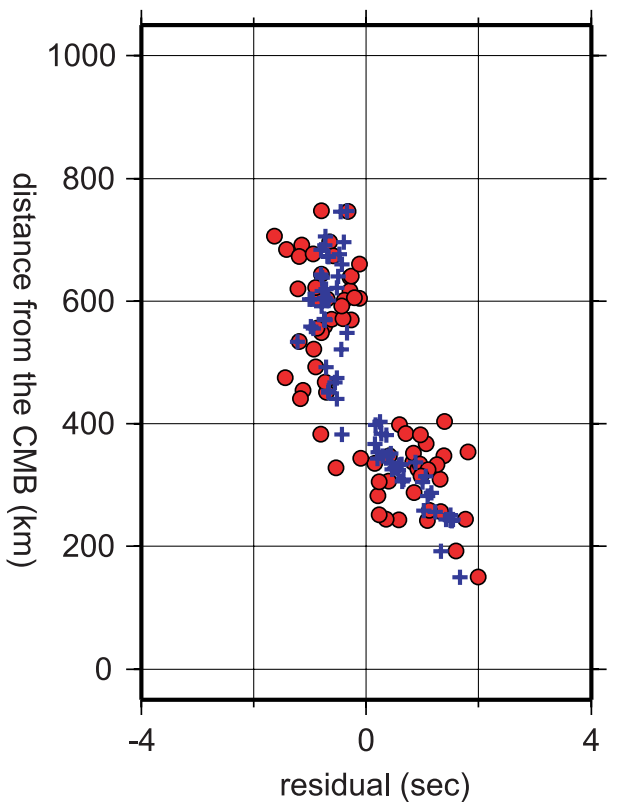

Figure 3. (a) Our preferred models when we assume the geographical boundary is located at $139^{\circ} \mathrm{E}$ (Model A; shown in green), at $140.5^{\circ} \mathrm{E}$ (Model B; shown in red), and at $142^{\circ} \mathrm{E}$ (Model C; shown in blue). The model for the eastern region (the LLVP region) and that for the western region are shown by solid and broken lines, respectively. (b) The E-W cross section of Model B. (c) The observed $S$ and $s S$ travel time residuals (red circles) and synthetic residuals for Model B (blue crosses) as functions of bottoming depth. Bottoming depths are computed for the IASP91 model.

are no more than about $100 \mathrm{~km}$ apart from each other. Thus these observations indicate the existence of small-scale heterogeneities with $100 \mathrm{~km}$ scale inside the LLVP.

[15] The small-scale heterogeneities are independently suggested by the $S c S-S$ travel times extracted from the observed waveforms. Figure $4 \mathrm{~b}$ shows the observed displacement seismograms at the KMI for the above four events. Because of the larger epicentral distances $\left(88.2^{\circ}-\right.$ $89.1^{\circ}$ ), the $S$ and $S c S$ are contaminated. They are most highly contaminated for event 020819 due to the larger magnitude $\left(M_{\mathrm{w}}=7.7\right)$ compared to the other three $\left(M_{\mathrm{w}}=\right.$ $6.3, M_{\mathrm{w}}=6.2$, and $M_{\mathrm{w}}=6.0$, respectively). We thus cannot accurately measure $S$ and $S c S$ travel times directly from the observed seismograms.

[16] Here we try to separate the $S$ and $S c S$ wavepackets from the contaminated displacement seismogram by assuming that the $S$ waveform of the KMI is identical to that of the QIZ. Under this assumption, we can estimate the $S c S$ of the KMI by subtracting the seismograms of the QIZ (or the $S$ of the KMI) from the seismograms of the KMI (or the $S$ and $S c S$ of the KMI). Note that, because the QIZ is located at smaller distances $\left(79.7^{\circ}-80.6^{\circ}\right)$, the $S$ arrives sufficiently earlier than the $S c S$ (by more than 20 seconds in our observations) and is considered to be well isolated. Figure $4 \mathrm{c}$ shows the plausibility of our method. The differential seismogram for event 020819 (blue in the top plot) between the seismograms of the KMI (red) and the QIZ (green) show a clear wavepacket, which has a peak about $8 \mathrm{~s}$ after the peak of the $S$. The obtained $S c S$ (blue in the bottom plot) has an almost identical waveform to that of the assumed
$S$ (or the $S$ wavepacket of the QIZ; green). We could not expect such similarity if our assumption is inappropriate.

[17] The obtained $S c S-S$ travel times are $8.0 \mathrm{~s}, 5.1 \mathrm{~s}, 5.6 \mathrm{~s}$, and $5.0 \mathrm{~s}$ for 020819, 021004, 021022, and 040111, respectively. The travel time for 020819 can be roughly confirmed in the velocity seismogram (Figure 4d), and travel times for the other three can be roughly confirmed in the displacement seismograms (the lower three in Figure $4 \mathrm{~b}$ ). This intuitively shows the robustness of our travel time measurements. Event 020819 has larger travel times for 2-3 s compared to the other three events, which shows the abrupt change in $S c S-S$ travel time residuals. These results also indicate the existence of small-scale heterogeneities inside the LLVP.

\section{Conclusion and Future Directions}

[18] In this study, we analyzed travel time and waveform data from the Vietnamese broadband seismograph array. The observed data suggest that the western geographical boundary is located around $140^{\circ} \mathrm{E}$ and that the strong lowvelocity region is confined to within $400 \mathrm{~km}$ from the CMB. They also suggest a sharp side edge (more than $4 \%$ velocity contrast within $200 \mathrm{~km}$ distance) and the existence of lateral heterogeneities with a $100 \mathrm{~km}$ scale. It is notable that both of sharp side edges and small-scale heterogeneities are observed in the African LLVP [e.g., Ni and Helmberger, 2003], which might indicate that the Pacific and the African LLVPs have the same cause of the heterogeneities. 
(a)

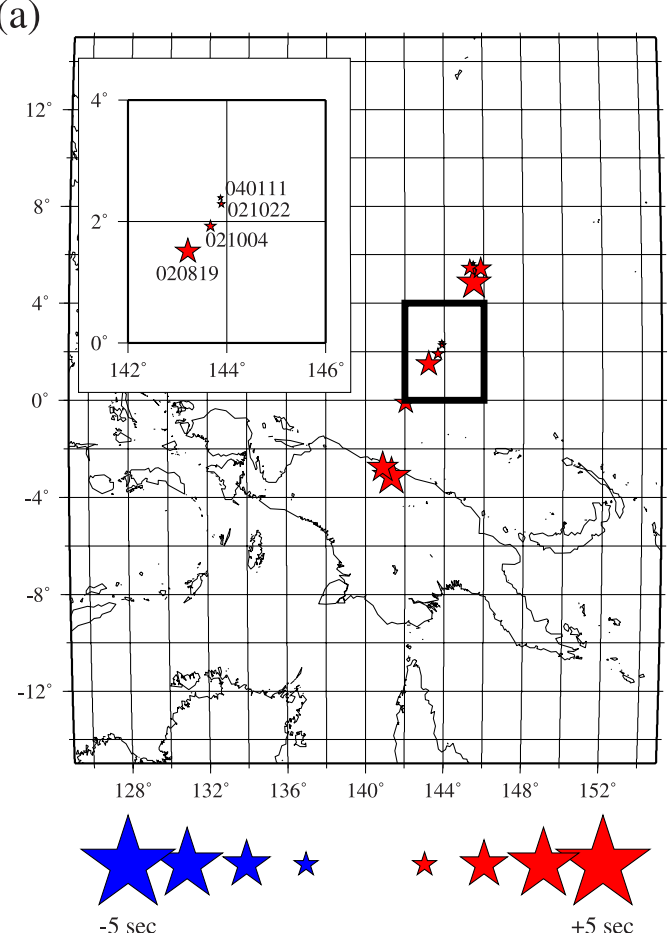

(b)

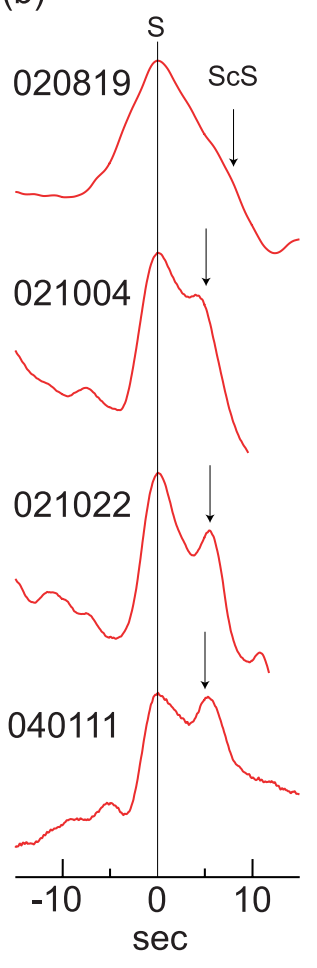

(c)
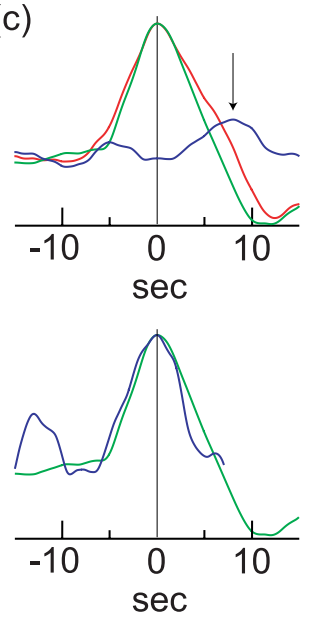

(d)

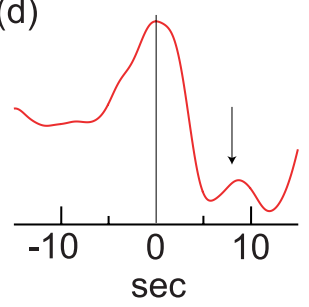

Figure 4. (a) Travel time residual data of $S$ whose bottoming point is within $300 \mathrm{~km}$ from the CMB. The residual is projected at the bottoming point and is plotted in the same manner as in Figure 2. At the top left is an enlargement of the region surrounded by thick black lines. The labels show the event date for the nearby residual data. (b) The observed displacement seismograms of $S$ and $S c S$ recorded at the KMI for the four events in Figure 4a. The arrows in Figures $4 \mathrm{~b}-4 \mathrm{~d}$ show the identified relative $S c S$ travel times to $S$. (c) The top plot shows the observed displacement $S$ and $S c S$ seismogram of the KMI (shown in red), the $S$ seismogram of the QIZ (shown in green), and their differential seismogram (shown in blue; assumed to be $S C S$ of the KMI) for event 020819. The bottom plot compares the $S$ of the QIZ (shown in green) and the estimated $S c S$ of the KMI (shown in blue). The times and the amplitudes are corrected for the comparison. (d) The observed velocity seismogram of the KMI. A causal filter with a corner frequency of $1 / 5 \mathrm{~Hz}$ is applied.

[19] For further constraint of the velocity structure at the western part of the Pacific LLVP, it will be crucial to use other kinds of data sets. For the central Pacific region, a few studies indicate the detailed structure of the Pacific LLVP. Ritsema et al. [1997] constrained the velocity gradient by using SV/SKS amplitude ratios as well as $S H-S K S$ travel times, and they suggested the strong negative velocity gradients (up to $3 \%$ velocity reductions) in the lowermost $190 \mathrm{~km}$. Lay et al. [2006] analyzed the precursors of ScS and suggested a weak negative top boundary $(-0.5$ to $-0.7 \%$ ) at $250-450 \mathrm{~km}$ from the CMB as well as several discontinuities inside the LLVP. It is not necessarily easy to definitely conclude that these features are not the case for the western part of the Pacific LLVP. After abundant data became available, we hope to test these features and discuss the regional and common features of the Pacific LLVP.

\section{References}

He, Y. M., L. X. Wen, and T. Y. Zheng (2006), Geographic boundary and shear wave velocity structure of the "Pacific anomaly" near the coremantle boundary beneath western Pacific, Earth Planet. Sci. Lett., 244, $302-314$.

Kennett, B. L. N., and E. R. Engdahl (1991), Traveltimes for global earthquake location and phase identification, Geophys. J. Int., 105, 429-465.
Lay, T., J. Hernlund, E. J. Garnero, and M. S. Thorne (2006), A postperovskite lens and $\mathrm{D}^{\prime \prime}$ heat flux beneath the central Pacific, Science, 314, $1272-1276$.

Ni, S. D., and D. V. Helmberger (2003), Seismological constraints on the South African superplume; could be the oldest distinct structure on Earth, Earth Planet. Sci. Lett., 206, 119-131.

Ni, S. D., E. Tan, M. Gurnis, and D. V. Helmberger (2002), Sharp sides to the African superplume, Science, 296, 1850-1852.

Ritsema, J., E. Garnero, and T. Lay (1997), A strong negative shear velocity gradient and lateral variability in the lowermost mantle beneath the Pacific, J. Geophys. Res., 102, 20,395-20,411.

Ritsema, J., S. D. Ni, and D. V. Helmberger (1998), Evidence for strong shear velocity reductions and velocity gradients in the lower mantle beneath Africa, Geophys. Res. Lett., 25, 4245-4248.

Takeuchi, N. (2007), Whole mantle SH velocity model constrained by waveform inversion based on three-dimensional Born kernels, Geophys. J. Int., 169, 1153-1163.

To, A., B. Romanowicz, and Y. Capdeville (2005), Constraints on lateral $S$ wave velocity gradient in $\mathrm{D}^{\prime \prime}$ region, Visual Geosci., 11, 13-40.

Wysession, M. E., L. Bartkó, and J. B. Wilson (1994), Mapping the lowermost mantle using core-reflected shear waves, J. Geophys. Res., 99, $13,667-13,684$.

Y. Morita and N. Takeuchi, Earthquake Research Institute, University of Tokyo, Yayoi 1-1-1, Bunkyo-ku, Tokyo 113-0032, Japan. (takeuchi@eri. u-tokyo.ac.jp)

N. D. Xuyen and N. Q. Zung, Institute of Geophysics, Vietnamese Academy for Science and Technology, 18 Hoang Quoc Viet Str., Cau Giay, Hanoi, Vietnam. 